\title{
The MAK-commission: finding solutions to society's future challenges
}

\author{
Jan G. Hengstler ${ }^{1}$
}

Received: 17 September 2018 / Accepted: 19 September 2018 / Published online: 24 September 2018

c) Springer-Verlag GmbH Germany, part of Springer Nature 2018

\section{Introduction}

Chemicals are essential to modern societies, and when properly used, substantially improve both health and quality of life. However, the consequences of unrecognized chemical risks may prove fatal. One well-known example is the high bladder cancer mortality in workers exposed to aromatic amines (Lukas et al. 2017). A second is the asbestos catastrophe, which spanned over a century (Gebel et al. 2014). Here, hundreds of thousands of mesothelioma cases could have been prevented with adequate toxicological research. Both examples illustrate the necessity to identify toxic risks before humans are exposed to potentially harmful compounds. It was within this context that the DFG Senate Commission for the Investigation of Health Hazards of Chemical Compounds in the Work Area (MAK-commission) was formed and has since done an outstanding job in curtailing risk.

The MAK-commission gathers together experts in the field of chemical risk assessment with the singular goal to identify possible risks in occupational environments. An important activity is to derive threshold values for specific chemicals at workplaces and to develop guidelines to prevent adverse consequences on human health. To date, more than 400 workplace chemicals have been evaluated. The established MAK-values are then re-evaluated as soon as new scientific knowledge about a particular compound is published in peer-reviewed journals. More importantly, the MAKcommission has developed the scientific concepts and principles that define how occupational exposure limits should be derived. For instance, scientific guidelines have been published for endpoints, such as sensory irritation (Brüning et al. 2014), or the categories for the derivation of MAK values for carcinogenic chemicals. Today, the commission

Jan G. Hengstler

hengstler@ifado.de

1 IfADo, Leibniz Research Centre for Working Environment and Human Factors at TU Dortmund, Ardeystr. 67, 44139 Dortmund, Germany is a world's leading organization for the establishment of threshold values at workplaces.

\section{The scientific principle}

The procedure for deriving occupational threshold values has been comprehensively described and made publicly available by the commission (http://www.dfg.de/en/dfg profile/statutory_bodies/senate/health_hazards/structure/ working_groups/derivation_mak/index.html). The derived MAK-value represents the maximum permissible concentration of a particular substance in the air of workplaces that does not cause adverse health effects. The value is established for long-term exposures of 8-h work days and 40-h work weeks. Where possible, threshold values are based on effects observed in humans using data generated from several sources, including occupational, medical and epidemiological studies. In addition, many threshold values have been directly derived from studies exposing volunteers under controlled conditions. However, the point of departure for most chemicals has been derived from animal experiments. Here, dose dependent exposures are performed, usually for at least 90 days, to identify the lowest dose that causes adverse effects [as well as the "no observed adverse effect level' (NOAEL)].

Several factors add to the complexity of establishing MAK-values. One example is that only data obtained from animal experiments with oral dosing are available for extrapolation to inhaled concentrations of the substance at the workplace. Specific rules have been established for this type of extrapolation. Importantly, the ruleset established by the MAK-commission also considers the mechanism of action of the evaluated compound. Depending on the type of compound-a carcinogen, sensitizer, one that causes risks during pregnancy, or causes germ cell mutagenicity-different, but precisely defined procedures are applied. Moreover, special consideration is given to chemicals that undergo percutaneous absorption, as they may contribute to systemic toxicity. These tasks are performed in working groups where 
scientific experts in their respective field, for example dermatologists, are recruited. According to the considerations of these working groups, labels (e.g., $\mathrm{H}$ for skin penetration) are added to the MAK-value.

An important aspect to consider during the establishment of limit values is that a MAK-value represents an 8-h time-weighted average. However, exposure to chemicals at workplaces may vary considerably during a working day. Therefore, the MAK-commission has established a ruleset under which short-term peak concentrations are acceptable. In the case of systemic toxicity, the half-life of the compound mainly determines the excursion factor. For local effects, such as irritation, human data are used to identify a concentration that acutely does not elicit any type of irritation. Accordingly, the excursion factor is specified. This not only considers the factor by which short-term peak concentrations exceed the MAK-value, but also regulates the minimum period that is allowed between individual exposure peaks, as well as the maximally acceptable duration of these individual excursions.

The ruleset of the MAK-commission considers a number of further aspects, all of which cannot be aptly summarized in the present article. However, one example which illustrates the complexity of establishing threshold values questions the course of action to be taken if a compound does not compromise health, but has an unpleasant odor. To address this, the MAK-commission established rules to objectify when an unpleasant smell is strong enough to justify limit values. The system of the MAK-commission is generally accepted as the most progressive and reliable ruleset worldwide and plays a key role in protecting humans from possible harmful effects of chemicals at workplaces. Based on these ruleset, standardized criteria documents are written, approved by the MAK-commission, subjected to public consultation and finally published. For the last couple of years, a collection of these documents has been made openly available online (http://onlinelibrary.wiley.com/book/https://doi. org/10.1002/3527600418/topics; MAK Collection with all substances online).

\section{History and future challenges}

The MAK-commission was founded in 1965, and under the leadership of Dietrich Henschler became recognized as a dynamic, internationally respected, and scientifically successful organization (Henschler 1985). His successor, Helmut Greim headed the commission between 1992 and 2007 , and was faced with the initial challenge that the MAK list contained a substantial number of traditional values for which documentation was missing-a gap that was rapidly filled. During his time as director, many principles of establishing scientific exposure limit values for chemicals were established and scientifically validated.

Helmut Greim's research clearly improved the measures taken to protect employees at their workplace. One key achievement of his pioneering work is that occupational threshold values now have to be derived according to a fixed ruleset. This guarantees that all chemicals at workplaces are regulated by the same principles, thus strongly reducing arbitrariness. Currently in his 80 s and officially retired, Helmut Greim still remains active scientifically, and continues to support the MAK-commission in his capacity as a consultant and guest scientist. His successor as chairperson, Andrea Hartwig, faces numerous scientific and societal challenges. Today, more than 100,000 chemicals are produced at a volume that exceeds a ton per year (Leist et al. 2017; Hengstler et al. 2006). The entry of new compounds and compound classes into production processes increases almost exponentially. This poses enormous challenges for regulatory toxicology, because this area of research should keep up with the current advances in material sciences and chemistry to allow preventive action. Circumstances, where for example, toxicological research occurs too slowly to fully characterize potential risks of compounds, should be avoided. There are already bitter lessons to be learnt from the catastrophes caused by asbestos and aromatic amines.

Besides the demanding scientific tasks, societal challenges have increased over the past decade. It is generally accepted that limit values for chemicals should be based on a fixed ruleset. However, it is a well-known phenomenon that exceptions may seem acceptable once individual interests are at play. MAK-values may have a major impact on the profit generated by a production process. For instance, a stricter limit value may cost millions or even billions of Euros for additional safety measures to be implemented if a compound is produced on a large scale. The conventional conflict of interest is that companies may tend to trivialize risks of chemicals to maximize their profits. However, the situation has become more complex in the past decades, with scenarios where companies and producer groups formed by the chemical industry support campaigns in the name of consumer and environmental protection that ultimately compromise the products of competitors, allowing them to maximize their own profits (Hengstler 2014). Moreover, NGOs and political parties tend to start campaigns that focus on specific individual compounds whipping up a media frenzy, most likely with the purpose to win followers and raise funds. This action may trigger a process that leads to scientifically unjustified banning of individual substances, while simultaneously overlooking others that in reality pose a higher risk to the population.

With these scientific and societal challenges, the MAKcommission fulfils the extremely important and certainly demanding task of guaranteeing a scientific, rule-based 
derivation of limit values. Consequently, the MAK-commission tackles one of the major future challenges faced by our society, which is to enjoy the advantages and comforts enabled by the use of chemicals and toxic working materials, while simultaneously minimizing the adverse consequences for human health.

\section{Compliance with ethical standards}

Conflict of interest Jan Hengstler worked in an honorary capacity (unpaid) as ad hoc expert for the MAK-commission.

\section{References}

Brüning T, Bartsch R, Bolt HM, Triebig G, Westphal G, van Thriel C (2014) Sensory irritation as a basis for setting occupational exposure limits. Arch Toxicol 88(10):1855-1879
Gebel T, Foth H, Damm G, Weiss C, Wollin KM, Hengstler JG (2014) Manufactured nanomaterials: categorization and approaches to hazard assessment. Arch Toxicol 88(12):2191-2211

Hengstler JG (2014) Letter to the editor. When science becomes fiction. A reply to "Do Toxic editors Trivialize Hidden Hazards?" Lab Times 5/2014a, 45-46, 2014b. http://www.labtimes.org/lette rs/Hengstler_Letter.pdf

Hengstler JG, Foth H, Kahl R, Kramer PJ, Lilienblum W, Schulz T, Schweinfurth H (2006) The REACH concept and its impact on toxicological sciences. Toxicology 220(2-3):232-239

Henschler D (1985) Development of occupational limits in Europe. Ann Am Conf Ind Hyg 12:37-40

Leist M, Ghallab A, Graepel R, Kroese D, van de Water B, Hengstler JG (2017) Adverse outcome pathways: opportunities, limitations and open questions. Arch Toxicol 91(11):3477-3505

Lukas C, Selinski S, Prager HM, Blaszkewicz M, Hengstler JG, Golka K (2017) Occupational bladder cancer: polymorphisms of xenobiotic metabolizing enzymes, exposures, and prognosis. J Toxicol Environ Health A 80(7-8):439-452 